Case Report

\title{
Hereditary Xerocytosis due to Mutations in PIEZO1 Gene Associated with Heterozygous Pyruvate Kinase Deficiency and Beta-Thalassemia Trait in Two Unrelated Families
}

\author{
Elisa Fermo, ${ }^{1}$ Cristina Vercellati, ${ }^{1}$ Anna Paola Marcello, ${ }^{1}$ Anna Zaninoni, ${ }^{1}$ \\ Richard van Wijk, ${ }^{2}$ Nadia Mirra, ${ }^{3}$ Cristina Curcio, ${ }^{4}$ Agostino Cortelezzi, ${ }^{1,5}$ \\ Alberto Zanella, ${ }^{1}$ Wilma Barcellini, ${ }^{1}$ and Paola Bianchi ${ }^{1}$ \\ ${ }^{1}$ U.O.C. Oncoematologia, U.O.S. Fisiopatologia delle Anemie, Fondazione IRCCS Ca' Granda Ospedale Maggiore Policlinico, \\ Milano, Italy \\ ${ }^{2}$ Department of Clinical Chemistry and Haematology, University Medical Center Utrecht, Utrecht, Netherlands \\ ${ }^{3}$ U.O.C. Pronto Soccorso, Pediatria Ambulatoriale e DH/MAC, Fondazione IRCCS Ca' Granda Ospedale Maggiore Policlinico, \\ Milano, Italy \\ ${ }^{4}$ U.O.S.D. Genetica Medica, Fondazione IRCCS Ca' Granda Ospedale Maggiore Policlinico, Milano, Italy \\ ${ }^{5}$ Universita degli Studi di Milano, Milano, Italy
}

Correspondence should be addressed to Elisa Fermo; elisafermo@yahoo.it

Received 14 October 2016; Accepted 15 February 2017; Published 6 March 2017

Academic Editor: Ramon Tiu

Copyright (C) 2017 Elisa Fermo et al. This is an open access article distributed under the Creative Commons Attribution License, which permits unrestricted use, distribution, and reproduction in any medium, provided the original work is properly cited.

\begin{abstract}
Hereditary xerocytosis (HX) is a rare disorder caused by defects of RBC permeability, associated with haemolytic anaemia of variable degree and iron overload. It is sometimes misdiagnosed as hereditary spherocytosis or other congenital haemolytic anaemia. Splenectomy is contraindicated due to increased risk of thromboembolic complications. We report the clinical, haematological, and molecular characteristics of four patients from two unrelated Italian families affected by HX, associated with beta-thalassemia trait and heterozygous pyruvate kinase deficiency, respectively. Two patients had been splenectomised and displayed thrombotic episodes. All patients had iron overload in the absence of transfusion, two of them requiring iron chelation. The diagnosis of HX was confirmed by LoRRca Osmoscan analysis showing a left-shifted curve. PIEZO1 gene sequencing revealed the presence of mutation p.E2496ELE, showing that this is one of the most frequent mutations in this disease. The concomitant defects did not aggravate the clinical phenotype; however, in one patient, the initial diagnosis of pyruvate kinase deficiency delayed the correct diagnosis of HX for many years and resulted in splenectomy followed by thrombotic complications. The study underlines the importance of a precise diagnosis in HX, particularly in view of splenectomy, and the need of a molecular confirmation of suspected RBC enzymopathy.
\end{abstract}

\section{Introduction}

Dehydrated stomatocytosis, also known as hereditary xerocytosis (HX, OMIM 194380), is the most frequent variant of hereditary stomatocytoses, a group of rare disorders characterized by a leak of monovalent cations $\left(\mathrm{Na}^{+}\right.$and $\left.\mathrm{K}^{+}\right)$from the red blood cells (RBCs) [1,2]. In particular, in HX red cells a decreased intraerythrocytic $\mathrm{K}^{+}$and total cations content is not accompanied by a proportional net gain of sodium and water, ultimately resulting in erythrocyte dehydration [3]. HX patients usually present with mild to moderate chronic haemolytic anaemia and splenomegaly, increased reticulocyte count, and slight macrocytosis; moreover, erythrocyte mean corpuscular haemoglobin concentration (MCHC) is increased and erythrocyte osmotic fragility is decreased. Blood smears show variable numbers of stomatocytes, but usually less than $10 \%$ [1]; HX is characterized by a propensity to iron overload [4]. The majority of HX cases has a 
mild presentation and may be overlooked for many years or sometimes misdiagnosed as hereditary spherocytosis or other congenital haemolytic anaemia [1]. However, unlike hereditary spherocytosis in which it is highly beneficial, splenectomy is contraindicated in HX due to increased risk of thromboembolic complications [5-7].

Osmotic gradient ektacytometry, which measures red cell deformability, osmotic fragility, and cell hydration, is the gold standard test for the definitive diagnosis and typically shows a leftward shift of the bell-shaped curve $[1,8]$. Sixteen different mutations in PIEZO1 gene have been identified in 27 unrelated families with $\mathrm{HX}$, primarily located in the highly conserved $\mathrm{COOH}$ terminus of the protein [9-18]. Functional studies of HX-associated PIEZO1 mutations demonstrate a partial gain-of-function phenotype with many mutants demonstrating delayed inactivation [10, 11, 19], suggesting increased cation permeability that leads to HX erythrocyte dehydration.

In this paper we report the clinical, haematological, and molecular characterization of two unrelated Italian families with HX due to mutation p.E2496ELE in PIEZO1 gene associated with beta-thalassemia trait in one and heterozygous pyruvate kinase (PK) deficiency in the other. By reviewing the other cases reported in literature carrying the same mutation, we exclude a synergetic effect between HX and the concomitant disorders. In one patient the initial diagnosis of pyruvate kinase deficient haemolytic anaemia delayed the correct diagnosis of $\mathrm{HX}$ and resulted in splenectomy followed by thrombotic complications.

\section{Case Report}

2.1. Family A. Two siblings, born after uncomplicated pregnancy form unrelated parents, were studied (Figure 1); the father (AI.1) was reported to have chronic haemolytic anaemia of unknown origin. AII.1 displayed chronic haemolysis and jaundice since infancy; she was diagnosed with PK deficiency on the basis of decreased enzyme activity and splenectomised in 1979 at the age of 21. After splenectomy jaundice disappeared and $\mathrm{Hb}$ levels increased from 9.4 to $11 \mathrm{~g} / \mathrm{dL}$, two episodes of deep vein thrombosis (DVT) occurred, at the age of 26 and 54. Hyperferritinemia $(1162 \mathrm{ng} / \mathrm{ml})$ was documented at the age of 46 and one cycle of deferoxamine chelation was done for 3 months only because of intolerance; at the age of $55 \mathrm{~T} 2{ }^{*}$ MRI revealed marked liver iron overload $(R=0.51)$ and patient started deferasirox treatment.

AII.2 was first studied in 1992 at the age of 29 after his wife's first pregnancy interruption at 26 weeks of gestation for severe hydrops foetalis attributed to parvovirus B19 infection. On that occasion, $\mathrm{Hb}$ levels were $12.5 \mathrm{~g} / \mathrm{dL}$, reticulocytes were $316 \times 10^{9} / \mathrm{L}$, unconjugated bilirubin was $5.9 \mathrm{mg} / \mathrm{dL}$, and serum ferritin was $723 \mathrm{ng} / \mathrm{ml}$; PK activity was slightly decreased (10.2 IU/gHb, normal values $13.3 \pm 2.29$ ); however, because of family history he was diagnosed with PK deficiency. Physical examination revealed scleral jaundice and splenomegaly. He underwent cholecystectomy at the age of 30 . Both siblings
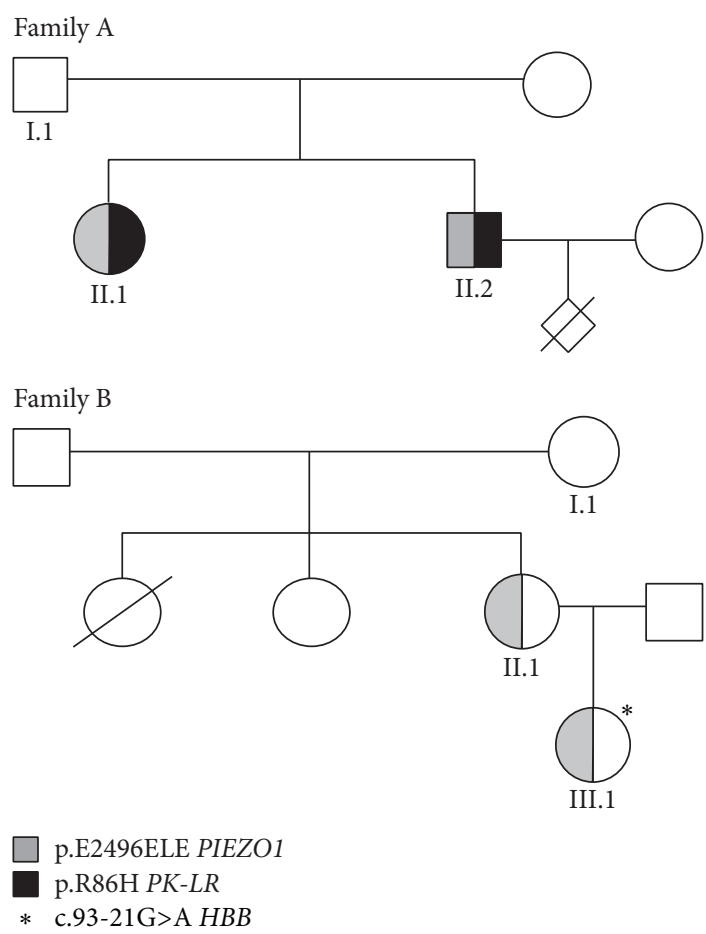

FIGURE 1: Genealogical trees of the reported families.

were referred to our Centre for reevaluation of haemolytic anaemia and iron overload. The patients' clinical and haematological data at the time of this study are shown in Table 1.

Both patients AII.1 and AII.2 displayed mild macrocytic anaemia and reticulocytosis, $13-15 \%$ stomatocytes in peripheral blood smear examination with $7 \%$ target cells, and $2-4 \%$ schistocytes, ovalocytes, and spherocytes (Figure 2). Increased serum ferritin levels were also detected in the absence of blood transfusions; HFE genotype was H63D/wt. Interestingly AII.1 showed mild spectrin deficiency with normal EMA binding test. RBC PK activity was decreased in both siblings (7 IU/gHb and $10.8 \mathrm{IU} / \mathrm{gHb}$, resp.). Direct sequencing of the entire coding region, intronic flanking regions, and promoter of $P K-L R$ gene showed heterozygosity for the c.257G $>$ A mutation (p.R86H). Because PK deficiency is an autosomal recessive disease this finding allowed excluding the previous diagnosis of PK deficiency and prompted us to investigate other causes of haemolysis in this family; the RBC morphology, the apparent dominant transmission, and the history of DVT in the splenectomised patient were suggestive of hereditary stomatocytosis.

LoRRca Osmoscan (Figure 3(a)) showed in AII.2 a left shift of the curve (Omin 156, n.v. 151-175; Ohyper 456, n.v. 473-534), indicating the presence of dehydrated red cells. In AII.1, a more pronounced left shift (Omin 119, Ohyper 428), was also associated with decreased EImax (0.541, n.v. 0.586-0.610), in line with the spectrin deficiency detected by SDS-PAGE analysis. PIEZO1 gene sequencing revealed in both siblings heterozygosity for the mutation c.7479_7484dupGGAGCT in exon 51 (p.E2496ELE), thus confirming the diagnosis of HX. 
TABLE 1: Clinical and haematological data of the patients at the time of the study.

\begin{tabular}{|c|c|c|c|c|c|}
\hline & AII. $^{\#}$ & AII.2 & BII. $^{\#}$ & BIII.1 & Normal values \\
\hline Sex & $\mathrm{F}$ & M & $\mathrm{F}$ & $\mathrm{F}$ & \\
\hline Age & 55 & 50 & 39 & 9 & \\
\hline Detection of anaemia & Infancy & $29 \mathrm{yrs}$ & $15 \mathrm{yrs}$ & Birth & \\
\hline Splenectomy (age) & Yes (21) & No & Yes (24) & No & \\
\hline Cholecystectomy (age) & No & Yes (30) & Yes (24) & No & \\
\hline Transfusions & No & No & No & No & \\
\hline $\mathrm{Hb}(\mathrm{g} / \mathrm{dL})$ & 11 & 13 & 12 & 10.8 & F 12-16; M 13.5-17.5 \\
\hline $\operatorname{MCV}(\mathrm{fl})$ & 108 & 103 & 100 & $66.4^{*}$ & $78-99$ \\
\hline $\mathrm{MCH}(\mathrm{pg})$ & 39.7 & 39.2 & 38.1 & 24.1 & $25-35$ \\
\hline $\mathrm{MCHC}(\mathrm{g} / \mathrm{dL})$ & 36.5 & 37.8 & 38 & 36.2 & $31-37$ \\
\hline Reticulocytes $\left(\times 10^{9} / \mathrm{L}\right)$ & 166 & 273 & 198 & 423 & $24-84$ \\
\hline Stomatocytes (\%) & 13 & 15 & 33 & 22 & \\
\hline PLTs $\left(\times 10^{9} / \mathrm{L}\right)$ & 696 & 172 & 875 & 329 & $130-400$ \\
\hline WBCs $\left(\times 10^{9} / \mathrm{L}\right)$ & 8.4 & 7.68 & 9.31 & 8.15 & $4.8-10.8$ \\
\hline Unconj bilirubin (mg/dL) & 1.33 & 4.82 & 1.33 & 2.8 & $<0.8$ \\
\hline Serum iron $(\mu \mathrm{g} / \mathrm{dL})$ & 101 & 150 & 167 & n.a. & $59-158$ \\
\hline Serum ferritin $(\mathrm{ng} / \mathrm{mL})$ & 745 & 1571 & 356 & 321 & F $15-150 ;$ M 30-400 \\
\hline Transferrin $(\mu \mathrm{g} / \mathrm{dL})$ & 149 & 170 & 237 & 221 & $200-360$ \\
\hline Transferrin saturation (\%) & 56 & 71 & 75 & n.a. & $16-54$ \\
\hline GLT (sec) & 180 & 68 & 480 & 195 & $23-45$ \\
\hline AGLT (sec) & $>900$ & $>900$ & $>900$ & $>900$ & $>900$ \\
\hline $\mathrm{NaCl}$ osmotic fragility & Reduced & Reduced & Reduced & Reduced & \\
\hline Pink test (\%) & 27 & 28 & 16 & 16 & $11-33$ \\
\hline EMA binding test & Normal & Normal & n.a. & n.a. & \\
\hline $\mathrm{Sp} / \mathrm{Bd} 3$ ratio & 0.90 & 1.14 & 1.11 & 1.05 & $0.97-1.19$ \\
\hline HFE genotype & $\mathrm{H} 63 \mathrm{D} / \mathrm{wt}$ & $\mathrm{H} 63 \mathrm{D} / \mathrm{wt}$ & $w t / w t$ & n.a. & wt/wt \\
\hline Gilbert genotype & $6 / 6$ & $6 / 6$ & $6 / 7$ & n.a. & $6 / 6$ \\
\hline PK activity (IU/gHb) & 7 & 10.8 & nd & nd & $11.9-16.1$ \\
\hline 2,3 DPG (nmol/gHb) & n.a. & 14183 & nd & nd & $10540 \pm 1720$ \\
\hline ATP & n.a. & 5674 & nd & nd & $4231 \pm 630$ \\
\hline$P K-L R$ genotype & c. $257 \mathrm{G}>\mathrm{A} / \mathrm{wt}$ & c. $257 \mathrm{G}>\mathrm{A} / \mathrm{wt}$ & nd & nd & \\
\hline
\end{tabular}

* $\beta$-Thalassemia trait; n.a. $=$ not available; $\mathrm{nd}=$ not determined.

${ }^{\text {\# }}$ Postsplenectomy.

2.2. Family B. Patient BII.1 was first recognized to be anaemic at the age of 15 years because of scleral jaundice; at the age of 23 in 1995 she was diagnosed with hereditary stomatocytosis based on the presence of stomatocytes at peripheral blood smear examination (12\%); in that occasion, she had Hb $11.9 \mathrm{~g} / \mathrm{dL}, \mathrm{MCV} 95 \mathrm{fL}$, reticulocytes $550 \times 10^{9} / \mathrm{L}$, serum ferritin $421 \mathrm{ng} / \mathrm{ml}$, transferrin saturation $70 \%$, and unconjugated bilirubin $6.7 \mathrm{mg} / \mathrm{dL}$. Abdominal ultrasound showed splenomegaly $(18 \times 12 \mathrm{~cm})$, hepatomegaly, and gallstones. Family study revealed in the mother (BI.1) a compensated haemolysis $\left(\mathrm{Hb} 12.6 \mathrm{~g} / \mathrm{dL}\right.$, reticulocytes $311 \times 10^{9} / \mathrm{L}$, and unconjugated bilirubin $2 \mathrm{mg} / \mathrm{dL}$ ) with $11 \%$ stomatocytes, suggesting dominant transmission of the disease.

One year later the patient underwent cholecystectomy for gallstones, associated with splenectomy. Surgery had no effect on $\mathrm{Hb}$ levels; postsplenectomy reticulocytes were 200$300 \times 10^{9} / \mathrm{L}$ and serum ferritin was $300-400 \mathrm{ng} / \mathrm{ml}$. At the age of 30 years, the patient became pregnant and she gave birth to a girl (BIII.1); pregnancy was complicated by gestosis, with delivery at 35 weeks. At birth the child was admitted 

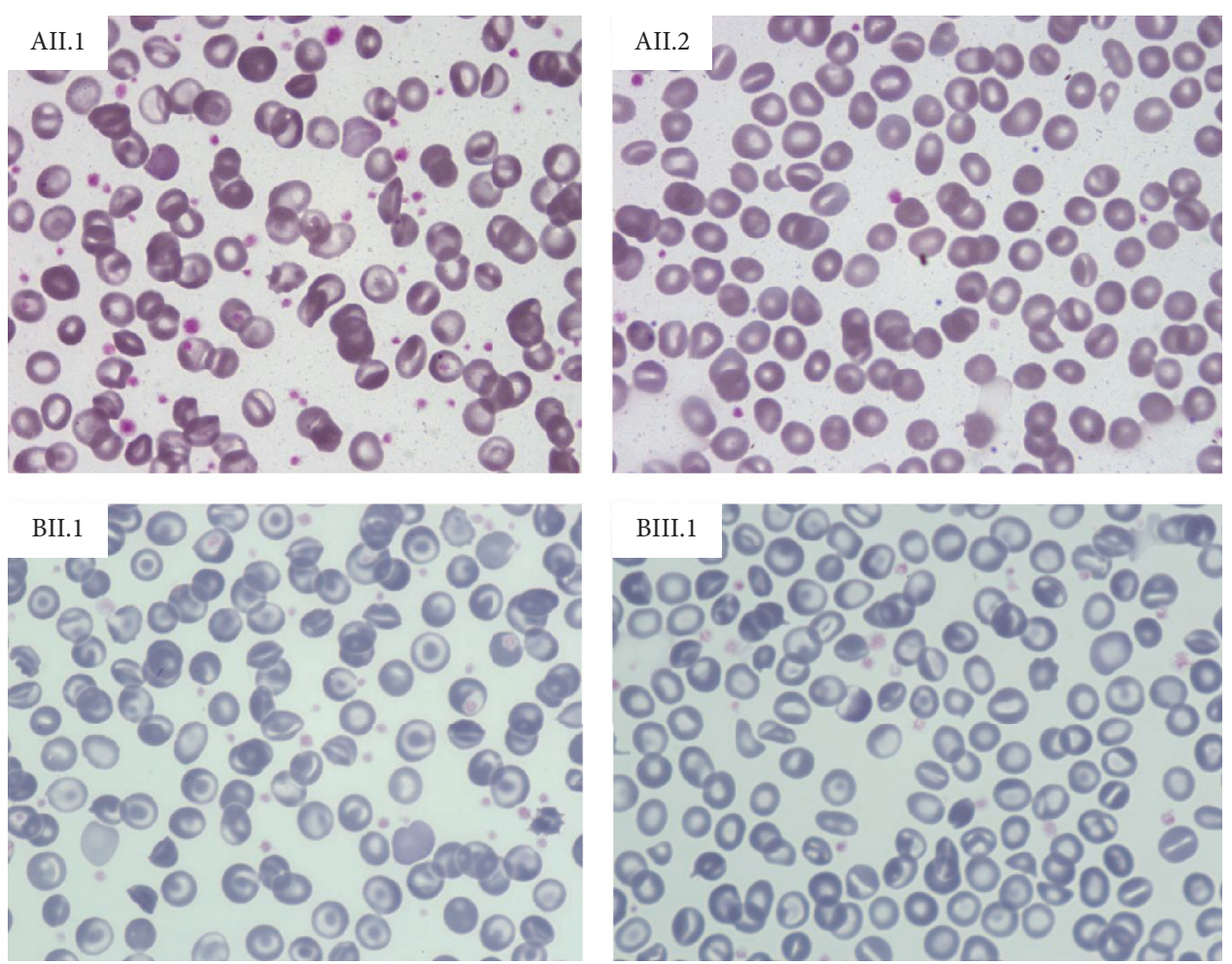

FIGURE 2: Light microscopy of peripheral blood, 63x.

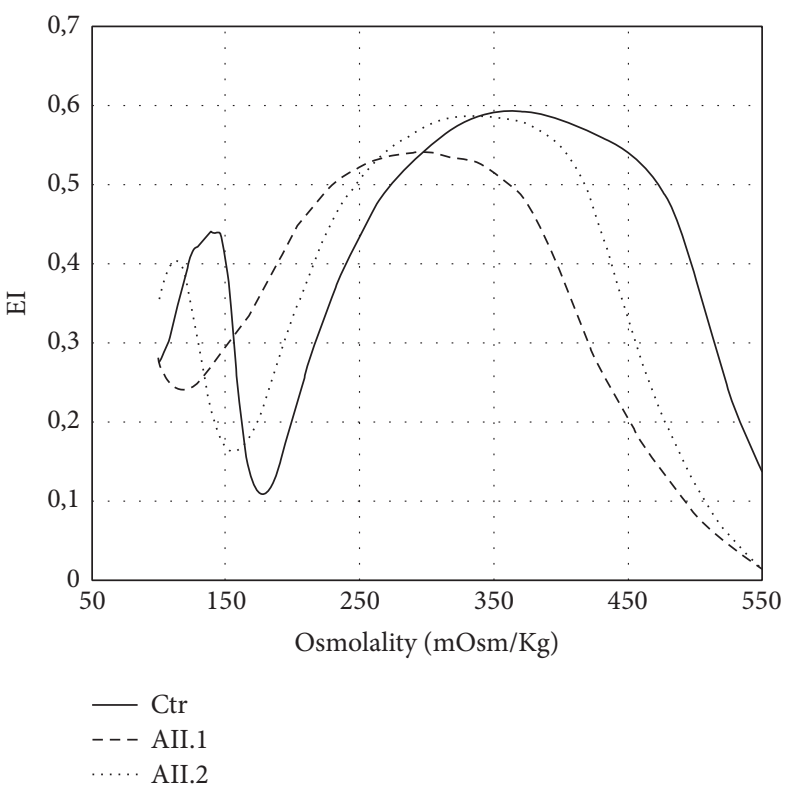

(a)

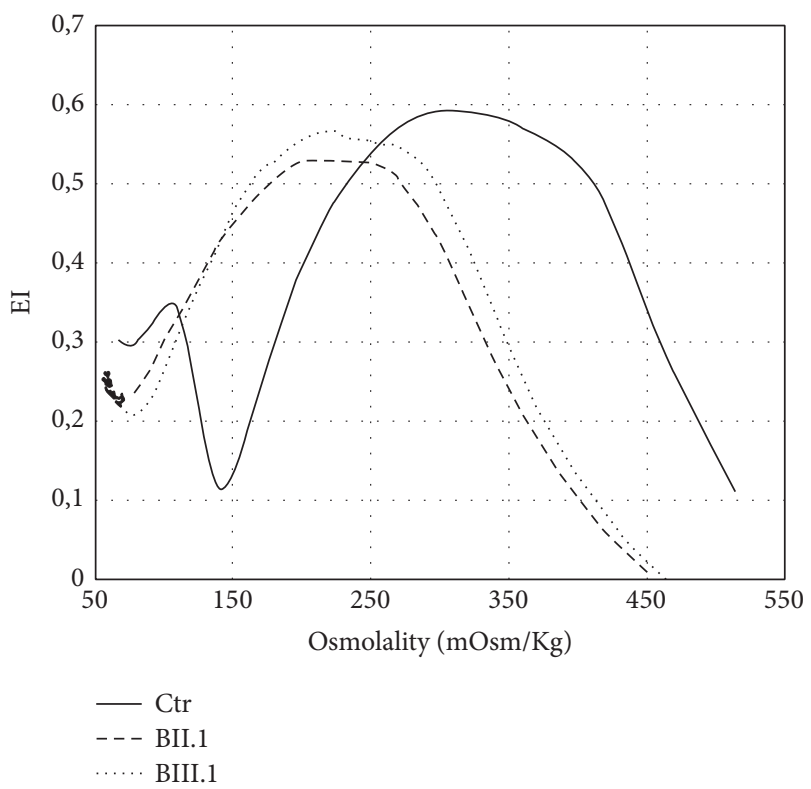

(b)

FIGURE 3: Results of LoRRca Osmoscan analysis in the affected patients compared to a normal control performed by Laser-Assisted Optical Rotation Cell Analyzer (LoRRca MaxSis, Mechatronics, Hoorn, The Netherlands). The osmotic gradient curves reflect RBC deformability as a continuous function of suspending medium osmolality. The following parameters were evaluated: EImax (maximal deformability, reflecting mean cellular surface area), Omin (osmolality at which deformability reaches its minimum, reflecting mean cellular surface-to-volume ratio), and Ohyper (the osmolality in the hypertonic region corresponding to $50 \%$ of the EImax, reflecting mean cellular hydration status). 
to the Intensive Care Unit for neonatal immaturity and respiratory distress, and she was diagnosed with hereditary stomatocytosis associated with beta-thalassemia trait $(\mathrm{Hb}$ $10.6 \mathrm{~g} / \mathrm{dL}, \mathrm{MCV} 71.9 \mathrm{fL}$, stomatocytes $22 \%$, reticulocytes 260 $\times 10^{9} / \mathrm{L}$, and unconjugated bilirubin $1.09 \mathrm{mg} / \mathrm{dL}$ ).

The mother and daughter were referred to our hospital for reevaluation of their anaemia and to confirm the diagnosis at molecular level. At the last follow-up patient BII.1 reported that a thromboembolic event occurred 20 years after splenectomy. Patient BII.1 displayed compensated haemolytic anaemia with $22 \%$ stomatocytes at peripheral blood smear examination, $13 \%$ target cells, and $2 \%$ schistocytes (Table 1 and Figure 2). Ferritin levels and transferrin saturation were increased in the presence of a normal HFE genotype. Bone marrow evaluation showed marked dyserythropoiesis. Case BIII.1 had mild microcytic anaemia, reticulocytosis, and increased ferritin levels; $17 \%$ of stomatocytes were detected at blood smear examination (Table 1 and Figure 2); the molecular testing for $H B B$ gene revealed the presence of the splicing mutation c.93-21G>A at heterozygote level, confirming the beta-thalassemia trait.

LoRRca Osmoscan (Figure 3(b)) showed the pronounced left shift of the curve typical of dehydrated stomatocytosis (Omin 71 and 76, resp., n.v. 136-151; Ohyper 342 and 352, n.v. 447-474). PIEZO1 gene sequencing revealed in both cases the presence of the mutation p.E2496ELE.

\section{Discussion}

There is an increasing interest in defects of RBC permeability due to the recent advances in knowledge of their molecular bases. Considered to be a group of very rare diseases, the number of reported cases of HX in the past three years is increased after the identification of the causative genes, such as PIEZO1 [9-11] and, more recently, KCNN4 codifying for Gardos channel [20-23].

The wide heterogeneity of clinical presentation and in particular the occurrence of mild/compensated cases may contribute to delay of the diagnosis $[14,16]$. Moreover, some patients displaying very few stomatocytes at the blood film examination may be misdiagnosed as hereditary spherocytosis or as suffering from an enzyme deficiency [1]. This was the case in patient AII.1, initially diagnosed as PK deficiency based on decreased enzyme activity, who underwent splenectomy and was eventually identified as HX with 30-year delay: the apparently dominant transmission of the anaemia, the absence of the expected increase of reticulocytes number after splenectomy, and $P K-L R$ genotyping showing monoallelic mutation ruled out the diagnosis of PK deficiency as a primary cause of haemolysis. This case therefore pinpoints the need of confirming a suspected RBC enzymopathy at the molecular level.

More than in other haemolytic anaemia, a precise diagnosis is of the utmost importance in HX in light of the increased risk of thrombotic complications following splenectomy [57] reported in all the splenectomised patients with PIEZO1 mutations but one. Patient AII.1 displayed two DVT episodes 5 and 33 years after splenectomy, and patient BII.1 had pulmonary embolism 20 years after surgery. It is worth mentioning that the only patient reported in the literature without thromboembolic complication after splenectomy had a relatively short follow-up [17].

Although usually well compensated and requiring no blood transfusions, dehydrated stomatocytosis is a heavily iron loading condition $[4,14]$, as also shown in our cases. The causes of this are not well established: haemolysis is commonly mild and it is unlikely to be per se the main cause of iron overload. An important factor in this regard may be dyserythropoiesis, which was also present in patient BII.1. The occurrence of dyserythropoiesis has been documented in a variant of hereditary stomatocytosis due to Gly796Arg mutation of the erythroid anion exchanger [24] and in one case with $\mathrm{HX}$ associated with hereditary high phosphatidylcholine haemolytic anaemia [17] but excluded in another PIEZO1 mutated patient described by Archer et al. [14].

HX has been long known to be a pleiotropic syndrome which combines in some families with haemolytic anaemia and perinatal edema; the latter, usually transient and of relatively benign prognosis [25-28], has been recently correlated with PIEZO1 mutation by Andolfo et al. [10]. The nonimmune foetal hydrops recorded in family $A$ is the most severe form so far described in patients with PIEZO1 mutations.

LoRRca Osmoscan proved to be very useful in orienting the diagnosis toward HX, later confirmed by the PIEZO1 gene analysis. Interestingly, patient AII.1 also displayed a decrease in EImax, as found in the case reported by GrootenboerMignot et al. [27]. In our patient the decrease in EImax is in line with the SDS-PAGE finding of a moderate spectrin deficiency. Spectrin deficiency in the absence of any mutation in RBC cytoskeletal proteins documented by whole exome sequencing has been reported in hereditary stomatocytosis due to KCNN4 mutation [23] and considered to be a secondary effect of a membrane perturbation $[29,30]$ disclosed by splenectomy [31].

All the four patients here described were heterozygous for mutation p.E2496ELE. The mutation was already reported in 8 unrelated cases described by Albuisson et al. [11] and very recently in a Japanese family [17] (Table 2). Although being also detected in 2 of 600 healthy French controls, this mutation was considered pathogenic; in fact, patch-clamp experiments in transfected HEK293 cells demonstrated a considerable increase in the inactivation time in the mutant compared to wild-type channel kinetics, indicating that it represents a gain-of-function mutation [11]. The finding of p.E2496ELE mutation also in our unrelated families (the first two families of Italian origin characterized at molecular level) supports its pathogenicity and confirms that this is one of the most frequent mutations in HX, with 15 patients so far reported in literature.

Combined defects of red cell membrane and/or metabolism are very rare, and the fact that carriership for a metabolic defect may be a modifier for the clinical expression of a membrane defect is still debated $[32,33]$; in particular, the copresence of beta-thalassemia trait seems to reduce the degree of haemolysis in patients with hereditary spherocytosis [34-37]. In the presented cases the clinical phenotype does not differ from the $\mathrm{HX}$ cases reported in literature 


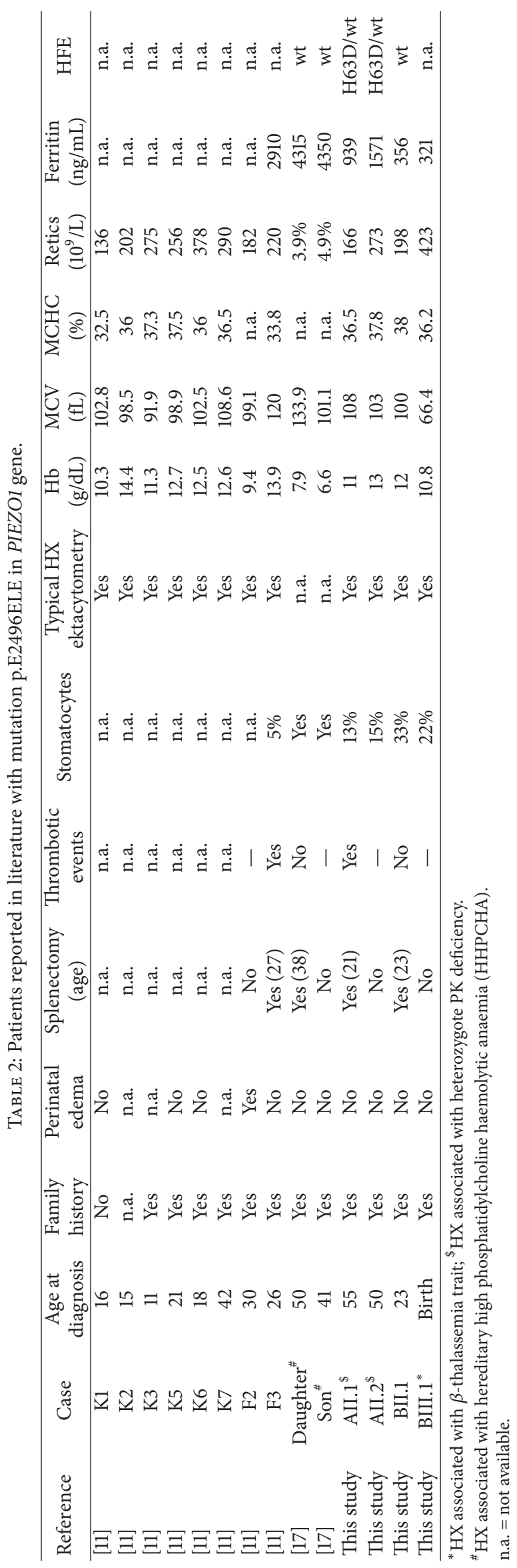


(Table 2), thus excluding a synergetic effect between HX and the concomitant disorders.

In conclusion, our data underline the importance of a precise diagnosis in HX, particularly in view of splenectomy because of the increased thrombotic risk, and the need of a molecular confirmation of a suspected RBC enzymopathy.

\section{Competing Interests}

The authors declare that there is no conflict of interests regarding the publication of this paper.

\section{Acknowledgments}

This study was supported by the Italian Ministry of Health: Ricerca Finalizzata Conv no. RF-2010-2303934.

\section{References}

[1] J. Delaunay, "The hereditary stomatocytoses: genetic disorders of the red cell membrane permeability to monovalent cations," Seminars in Hematology, vol. 41, no. 2, pp. 165-172, 2004.

[2] E. Glogowska and P. G. Gallagher, "Disorders of erythrocyte volume homeostasis," International Journal of Laboratory Hematology, vol. 37, no. 1, pp. 85-91, 2015.

[3] L. J. Bruce, "Hereditary stomatocytosis and cation leaky red cells-recent developments," Blood Cells, Molecules, and Diseases, vol. 42, no. 3, pp. 216-222, 2009.

[4] P.-Y. Syfuss, A. Ciupea, S. Brahimi et al., "Mild dehydrated hereditary stomatocytosis revealed by marked hepatosiderosis," Clinical and Laboratory Haematology, vol. 28, no. 4, pp. 270274, 2006.

[5] A. P. Marcello, C. Vercellati, E. Fermo et al., "A case of congenital red cell pyruvate kinase deficiency associated with hereditary stomatocytosis," Blood Cells, Molecules, and Diseases, vol. 41, no. 3, pp. 261-262, 2008.

[6] G. W. Stewart, J. A. L. Amess, S. W. Eber et al., "Thromboembolic disease after splenectomy for hereditary stomatocytosis," British Journal of Haematology, vol. 93, no. 2, pp. 303-310, 1996.

[7] X. Jaïs, S. J. Till, T. Cynober et al., "An extreme consequence of splenectomy in dehydrated hereditary stomatocytosis: gradual thrombo-embolic pulmonary hypertension and lung-heart transplantation," Hemoglobin, vol. 27, no. 3, pp. 139-147, 2003.

[8] L. Da Costa, J. Galimand, O. Fenneteau, and N. Mohandas, "Hereditary spherocytosis, elliptocytosis, and other red cell membrane disorders," Blood reviews, vol. 27, no. 4, pp. 167-178, 2013.

[9] R. Zarychanski, V. P. Schulz, B. L. Houston et al., "Mutations in the mechanotransduction protein PIEZO1 are associated with hereditary xerocytosis," Blood, vol. 120, no. 9, pp. 1908-1915, 2012.

[10] I. Andolfo, S. L. Alper, L. De Franceschi et al., "Multiple clinical forms of dehydrated hereditary stomatocytosis arise from mutations in PIEZO1," Blood, vol. 121, no. 19, pp. 39253935, 2013.

[11] J. Albuisson, S. E. Murthy, M. Bandell et al., "Dehydrated hereditary stomatocytosis linked to gain-of-function mutations in mechanically activated PIEZO1 ion channels," Nature Communications, vol. 4, article 1884, 2013.
[12] C. Beneteau, G. Thierry, S. Blesson et al., "Recurrent mutation in the PIEZO1 gene in two families of hereditary xerocytosis with fetal hydrops," Clinical Genetics, vol. 85, no. 3, pp. 293-295, 2014.

[13] B. E. Shmukler, D. H. Vandorpe, A. Rivera, M. Auerbach, C. Brugnara, and S. L. Alper, "Dehydrated stomatocytic anemia due to the heterozygous mutation $\mathrm{R} 2456 \mathrm{H}$ in the mechanosensitive cation channel PIEZO1: a case report," Blood Cells, Molecules, and Diseases, vol. 52, no. 1, pp. 53-54, 2014.

[14] N. M. Archer, B. E. Shmukler, I. Andolfo et al., "Hereditary xerocytosis revisited," American Journal of Hematology, vol. 89, no. 12, pp. 1142-1146, 2014.

[15] M. B. Sandberg, M. Nybo, H. Birgens, and H. Frederiksen, "Hereditary xerocytosis and familial haemolysis due to mutation in the PIEZO1 gene: a simple diagnostic approach," International Journal of Laboratory Hematology, vol. 36, no. 4, pp. e62-e65, 2014.

[16] M. Paessler and H. Hartung, "Dehydrated hereditary stomatocytosis masquerading as MDS," Blood, vol. 125, no. 11, p. 1841, 2015.

[17] S. Imashuku, H. Muramatsu, T. Sugihara et al., "PIEZO1 gene mutation in a Japanese family with hereditary high phosphatidylcholine hemolytic anemia and hemochromatosisinduced diabetes mellitus," International Journal of Hematology, vol. 104, no. 1, pp. 125-129, 2016.

[18] R. Del Orbe Barreto, B. Arrizabalaga, A. B. De la Hoz Rastrollo et al., "Hereditary xerocytosis, a misleading anemia," Annals of Hematology, vol. 95, no. 9, pp. 1545-1546, 2016.

[19] C. Bae, R. Gnanasambandam, C. Nicolai, F. Sachs, and P. A. Gottlieb, "Xerocytosis is caused by mutations that alter the kinetics of the mechanosensitive channel PIEZO1," Proceedings of the National Academy of Sciences of the United States of America, vol. 110, no. 12, pp. E1162-E1168, 2013.

[20] R. Rapetti-Mauss, C. Lacoste, V. Picard et al., "A mutation in the Gardos channel is associated with hereditary xerocytosis," Blood, vol. 126, no. 11, pp. 1273-1280, 2015.

[21] I. Andolfo, R. Russo, F. Manna et al., "Novel Gardos channel mutations linked to dehydrated hereditary stomatocytosis (xerocytosis)," American Journal of Hematology, vol. 90, no. 10, pp. 921-926, 2015.

[22] E. Glogowska, K. Lezon-Geyda, Y. Maksimova, V. P. Schulz, and P. G. Gallagher, "Mutations in the Gardos channel (KCNN4) are associated with hereditary xerocytosis," Blood, vol. 126, no. 11, pp. 1281-1284, 2015.

[23] E. Fermo, A. Y. Bogdanova, P. Petkova-Kirova et al., "Gardos channel mutation is associated with hereditary dehydrate stomatocytosis: a complex channelopathy," Blood, vol. 126, supplement 1, article 3333, 2015.

[24] A. Iolascon, L. De Falco, F. Borgese et al., "A novel erythroid anion exchange variant (Gly796Arg) of hereditary stomatocytosis associated with dyserythropoiesis," Haematologica, vol. 94, no. 8, pp. 1049-1059, 2009.

[25] S. Grootenboer, C. Barro, T. Cynober et al., "Dehydrated hereditary stomatocytosis: a cause of prenatal ascites," Prenatal Diagnosis, vol. 21, no. 13, pp. 1114-1118, 2001.

[26] S. Grootenboer, P. O. Schischmanoff, T. Cynober et al., "A genetic syndrome associating dehydrated hereditary stomatocytosis, pseudohyperkalaemia and perinatal oedema," British Journal of Haematology, vol. 103, no. 2, pp. 383-386, 1998.

[27] S. Grootenboer-Mignot, A. Crétien, I. Laurendeau et al., "Sublethal hydrops as a manifestation of dehydrated hereditary stomatocytosis in two consecutive pregnancies," Prenatal Diagnosis, vol. 23, no. 5, pp. 380-384, 2003. 
[28] M. Entezami, R. Becker, M. Marcinkowski, and H. T. Versmold, "Xerocytosis with concomitant intrauterine ascites: first description and therapeutic approach," Blood, vol. 87, no. 12, pp. 5392-5393, 1996.

[29] N. Fortier, L. M. Snyder, F. Garver, C. Kiefer, J. McKenney, and N. Mohandas, "The relationship between in vivo generated hemoglobin skeletal protein complex and increased red cell membrane rigidity," Blood, vol. 71, no. 5, pp. 1427-1431, 1988.

[30] M. Salomao, K. Chen, J. Villalobos, N. Mohandas, X. An, and J. A. Chasis, "Hereditary spherocytosis and hereditary elliptocytosis: aberrant protein sorting during erythroblast enucleation," Blood, vol. 116, no. 2, pp. 267-269, 2010.

[31] M. Mariani, W. Barcellini, C. Vercellati et al., "Clinical and hematologic features of 300 patients affected by hereditary spherocytosis grouped according to the type of the membrane protein defect," Haematologica, vol. 93, no. 9, pp. 1310-1317, 2008.

[32] C. Vercellati, A. P. Marcello, E. Fermo, W. Barcellini, A. Zanella, and P. Bianchi, "A case of hereditary spherocytosis misdiagnosed as pyruvate kinase deficient hemolytic anemia," Clinical Laboratory, vol. 59, no. 3-4, pp. 421-424, 2013.

[33] R. van Zwieten, B. A. van Oirschot, M. Veldthuis et al., "Partial pyruvate kinase deficiency aggravates the phenotypic expression of band 3 deficiency in a family with hereditary spherocytosis," American Journal of Hematology, vol. 90, no. 3, pp. E35-E39, 2015.

[34] M. K. Cihan, H. Gökçe, M. Oruç, and L. Olcay, "Hemolytic crisis as the initial presentation of hereditary spherocytosis induced by parvovirus B19 and herpes virus infection in a patient with the thalassemia trait: a case report," Turkish Journal of Hematology, vol. 29, no. 4, pp. 425-426, 2012.

[35] E. M. Del Giudice, S. Perrotta, B. Nobili, L. Pinto, L. Cutillo, and A. Iolascon, "Coexistence of hereditary spherocytosis (HS) due to band 3 deficiency and $\beta$-thalassaemia trait: partial correction of HS phenotype," British Journal of Haematology, vol. 85, no. 3, pp. 553-557, 1993.

[36] M. Aksoy and S. Erdem, "The Combination of Hereditary Spherocytosis and Heterozygous Beta-Thalassaemia," Acta Haematologica, vol. 39, no. 3, pp. 183-191, 1968.

[37] J. M. Andrien, A. Heusden, C. Lambotte, and J. Hugues, "[Hereditary spherocytosis and beta-thalassemia: coexistence of 2 genes in a Sicilian family]," Acta paediatrica Belgica, vol. 25, no. 1, pp. 35-43, 1971. 


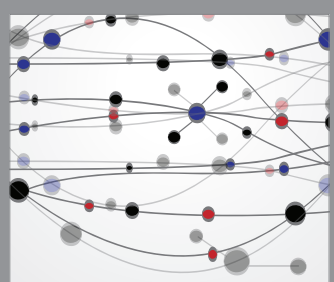

The Scientific World Journal
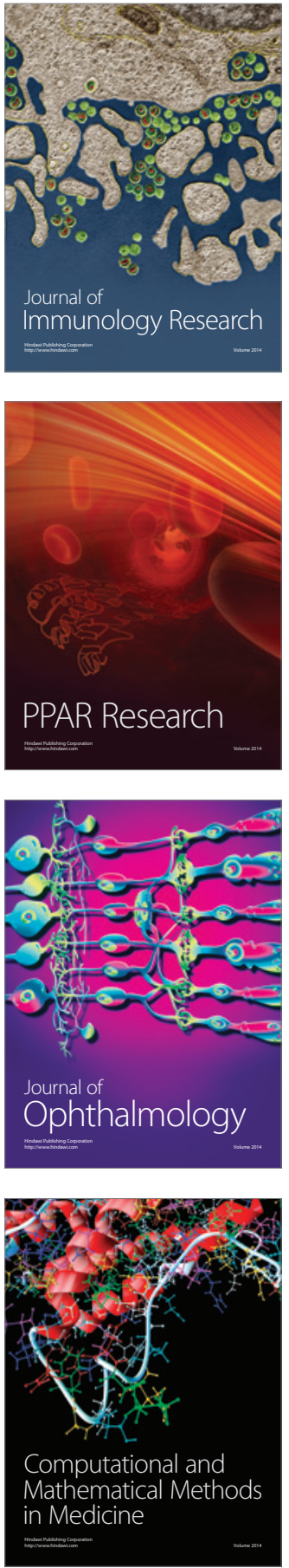

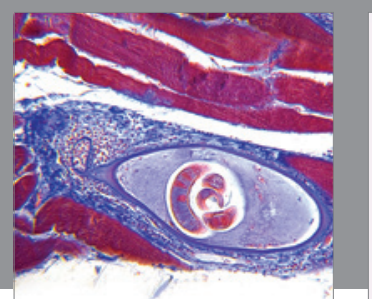

Gastroenterology Research and Practice
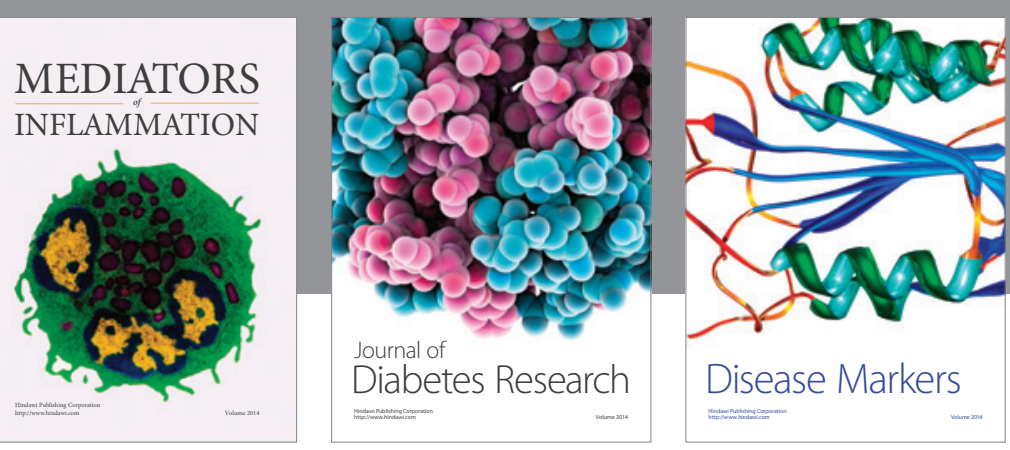

Disease Markers

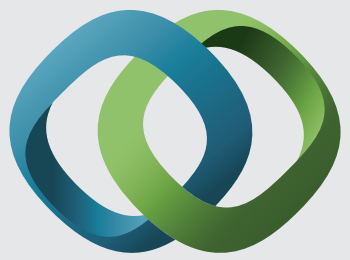

\section{Hindawi}

Submit your manuscripts at

https://www.hindawi.com
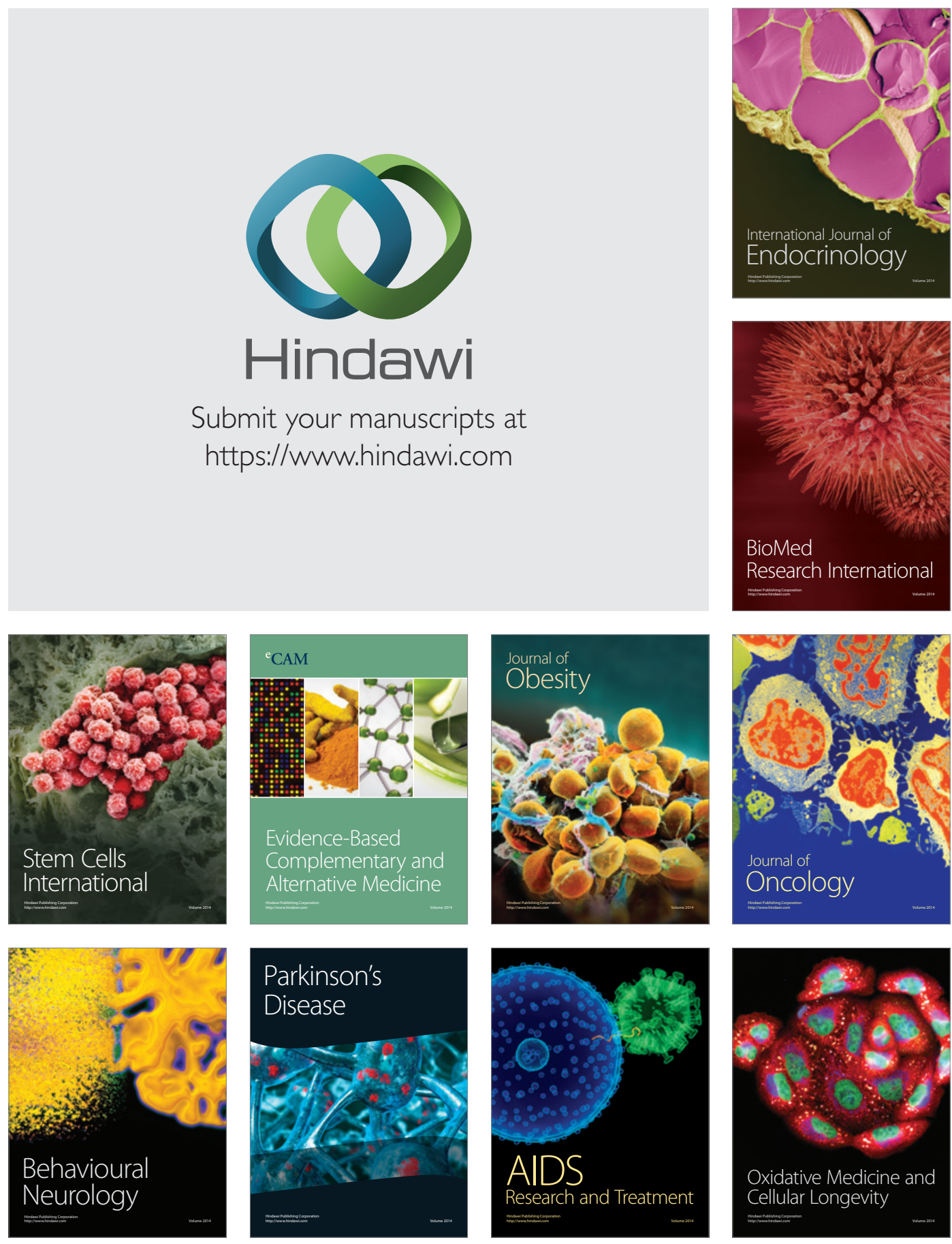